ஏலகிரி மலைவாழ் மக்களின் திருமணச் சடங்குமுறைகளில் வெளிப்படும் பண்பாட்டுக் கூறுகள்

வெ. திருவேணி அ, இரா. லோகநாதன் அ, *

அ தமிழ்ப் பண்பாட்டு மையம், அழகப்பா பல்கலைக்கழகம், காரைக்குடி-630003, தமிழ்நாடு, இந்தியா.

Cultural Elements Expressed in the Wedding Ceremonies of the Yelagiri Hill Tribes

\author{
V. Thiruveni a, R. Loganathan ${ }^{a}$ * \\ ${ }^{a}$ Centre for Tamil Culture, Alagappa University, Karaikudi-630003, Tamil Nadu, India
}

* Corresponding Author:

r.loganathantpt@gmail.com

Received: $24-08-2020$

Revised: 25-02-2021

Accepted: 28-02-2021

Published: 03-03-2021

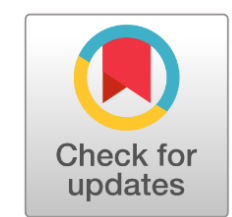

\begin{abstract}
Marriage is a kind of relationship that takes place in human life. A marriage takes place depending on the respective culture and tradition of a particular group of people living as a society. This is a process that takes place all over the world according to their circumstances. These circumstances reveal the prosperity of their cultural practices. In this case, the marriage ceremony of the tribes living in the Yelagiri hills of Tirupattur district depends on the old customs and traditions. This article goes to apply Richard M.Dorson's folk cultural aspect theory to know the methods and record the marriage system as an essential part of these tribes.
\end{abstract}

Keywords: Marriage, Human life, Marriage Ceremony, Cultural, Yelagiri Tribals.

\title{
ஆசிரியர் குறிப்பு
}

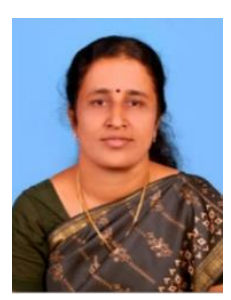

முனைவர் வெ. திருவேணி அவர்கள், இவர் அண்ணாமலைப் பல்கலைக்கழகத்தில் முதுகலை (எம்.ஏ.) பட்டமும் அழகப்பா பல்கலைக் கழகத்தில் விக்கிரமனின் “ராஜாதித்தன் சபதம்" பற்றி ஆராய்ச்சி செய்து ஆய்வியல் நிறைஞர் (எம்.பில்) பட்டமும் இதே பல்கலைக்கழகத்தில் 'கலைஞரின் புலப்பாட்டுத்திறன்' என்னும் தலைப்பில் ஆய்வுசெய்து முனைவர் (பி.எச்டி.) பட்டமும் பெற்றுள்ளார். அரிதின் முயன்று ஆழங்கால்பட ஆற்றியுள்ள இவ்வாய்வுகள். இவரது நுண்மாண் நுழைபுலத்திற்குரிய நுவலரும் சான்றுகள் ஆகும். தற்பொழுது அமுதத் தமிழ்மணக்கும் அழகப்பா பல்கலைக்கழகத் தொலைநிலைக்கல்வி இயக்ககத்தில் உதவிப் பேராசிரியராகப் பணிபுரிந்து வருகின்றார். இவரின் நெறியாள்கையின்கீழ் ஐந்துபேர் முனைவர் (பி.எச்டி.) பட்டமும். 15 பேர் ஆய்வியல் நிறைஞர் (எம்.பில்) பட்டமும் பெற்றுள்ளனர். பத்துக்கும் மேற்பட்ட நூல்களைப் படைத்துள்ளார்.

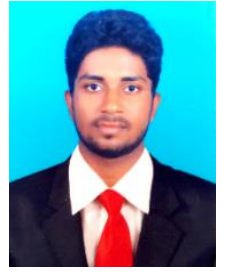

இரா. லோகநாதன் அவர்கள், இளங்கலைத் தமிழ் தூய நெஞ்சக் கல்லூரியிலும், முதுகலைத் தமிழ் புதுவை மத்தியப் பல்கலைக் கழகத்திலும், தற்போது முனைவர் பட்ட ஆய்வினை காரைக்குடி அழகப்பா பல்கலைக் கழகத்தில் ம மேற்கொள்கிறார். இவரின் தலைப்பானது ஏலகிரி மலைவாழ் மக்களின் வாழ்வியலும் பண்பாடும் என்பதாகும். 


\section{முன்னுரை}

திருமணம் மனிதனின் உள்ளம், உடல் சார்ந்த தேவைகளை நிறைவு செய்வதோடு மட்டுமின்றி, சமுதாயத்தில் பண்பாட்டு உறுப்பினராக இணைத்துக்கொள்ளவும் வகை செய்கிறது. திருமணம் என்பது ஆண்-பெண் இருவரும் ஒருவரோடு ஒருவர் இணைந்து இல்லறம் நடத்தும் பொருட்டு ஏற்படுத்திக் கொள்ளும் உடன்படிக்கையாகும். இதனால், மணம் என்பது மனிதனின் பாலியல் உந்துதல்களைச் சமுதாய ஒப்புதலுடன் நிறைவு செய்து கொள்ளும் பண்பாட்டு நிறுவனம் என்பதையும், அது சடங்குகளின்படி முன்பின் அறிமுகமில்லாதவர்களை இணைக்கும்போது உடன்படிக்கையாக அமைகிறது. இந்நிலையில், கிழக்குத் தொடர்ச்சி மலை வரிசையில் அமைந்துள்ள திருப்பத்தூர் மாவட்ட ஏலகிரி மலையில் வசிக்கும் இந்து மலையாளி, இருளர், காராளக்கவுண்டர் போன்ற இனக்குழு மக்களின் திருமண நிகழ்ச்சிகளில் இடம்பெறும் திருமணப் பாடல்கள் மற்றும் சடங்கு முறைகளைப் பண்பாட்டு ஆய்விற்கு உட்படுத்தும் விதமாக இக்கட்டுரை அமைகின்றது.

\section{முன் ஆய்வுகள்}

தமிழகப் பழங்குடி மக்களைப் பற்றி ஆய்வினை ஆய்வறிஞர்கள் மேற்கொண்டுள்ளனர். அவ்வகையில், க.ப. அறவாணனின் பழங்குடி மக்கள் பற்றிய ஆய்வுகள், மு. இராசேந்திரனின் மெக்கன்சி சுவடிகளில் தமிழகப் பழங்குடி மக்கள், ஆ.செல்லபெருமாளின் தென் தமிழ் நாட்டுப் பழங்குடிகள், தா.இராபர்ட்டின் சத்திய சோசப்பின் குரும்பர்களின் உறவுமுறை, தமிழ்நாடனின் மலைவாழ் பழங்குடி மக்கள், அண்ணாமலையின் சவ்வாதுமலை ஆய்வு போன்றவை குறிப்பிடத்தக்கன. மேலும், கா. ஆறுமுகத்தின் ஏலகிரி மலைவாழ் மக்களின் (மலையாளிகள்) வாழ்வியல், க. லோகநாதனின் ஏலகிரி மலையின் நாட்டுப்புறவியல் கூறுகள் போன்றவை இக்கட்டுரைக்கு ஆய்வு முன்னோடிகளாக அமைகின்றன (Thayammal Aravanan, 1987).

\section{ஆய்வு நோக்கம்}

நாட்டுப்புற மக்களின் வாழ்வியலை அடிப்படையாகக் கொண்டு நடத்தப்பெறும் ஆய்வுமுறையே நாட்டுப்புறப் பண்பாட்டுக் கோட்பாடு ஆகும். பண்பாட்டுக் கோட்பாட்டினைக் குறித்து ரிச்சர்டு எம்.டார்சன் குறிப்பிடுகையில், ஓர் இனத்தினரின் வாழ்க்கை முறை, பழக்க வழக்கங்கள் முதலியவற்றைக் கொண்டு அகப்பண்பாட்டினை ஆராயலாம்" (Richard M. Dorson, 1972, p.43) என்பார். இக்கருத்து நாட்டுப்புறவியலின் அனைத்துக் கூறுகளையும் தழுவியதாக அமைந்துள்ளு. அவ்வகையில், ஏலகிரி பழங்குடிகளிடையே திருமணச் சடங்கிற்குள் பொதிந்துள்ள பண்பாட்டுக் கூறுகளை வெளிக்கொணரும் நோக்கில் இக்கட்டுரை பயணிக்கிறது.

\section{திருமணம் - தோற்றமும் வளர்ச்சியும்}

"மணம்" என்ற சொல்லுக்கு அறிஞர் பலர், ஆய்வின் மூலம் பொருள் விளக்கம் அளித்துள்ளனர். எல்.எச்.மார்கன் (Morgan, 1877). "மனிதனின் வாழ்வுநிலை வளர்ச்சியில் தொடக்க கால கூட்டுச் சமுதாய அமைப்பில் (Primitive Society) கட்டுப்பாடு இல்லாப் புணர்ச்சி நிலவிய குழு மணத்தை முதல் மணம் என்றும் தொடர்ந்து பல பெண்களை ஓர் ஆடவர் மணம் செய்து கொள்ளுதலும் இறுதியாக ஒருவன் ஒருத்தியை மணந்து கொள்வதும் (Monogamy) சமுதாயத்தில் தோன்றி வளர்ந்தன". என்பார். இக்கூட்டிணைவு உணர்வுகள், உந்துதல்கள், கூடிவாழ்தல் என்ற நிலைகள் மாறி காலப்போக்கில் பல்வேறு சடங்குகளுக்குட்பட்டு ஒழுக்கமான முறைக்குள் ‘திருமணம்’ என்னும் அமைப்பின்வழி ஆக்கம் பெற்றது. மனித சமூகத்தில் மிகக் குறிப்பிடத்தக்க வளர்ச்சி கட்டம் திருமணம் என்பதாகும். இதன் பின்னரே உறவுமுறை பார்க்கும் வழக்கம் நிலைபெறத் தொடங்கி இருத்தல் வேண்டும்.

பண்டைக் காலத்தில் பொருளின்றிப் பல இடங்களில் அலைந்து திரிந்து வாழ்ந்தது முதல் நிலையாகும். பின்னர் கால்நடைகளை ஆடவர் உடைமையாகக் கொண்டனர். அடுத்ததாகப் பெண்களின் 
உடைமைகளாக நிலம் அமைந்தது. நிலச்சுவான்தார்களான பெண்களுக்கு நிலத்தைப் பயன்படுத்தவும் பயிர் செய்யவும் ஆள்துணை தேவைப்பட்டது. நிலவுடைமைக்கும் பாதுகாப்பிற்கும் ஆள் நிரந்தரத் தேவையாயிற்று. பின்னர் அத்துணை முதன்மையாகித் ‘திருமணம்’ என்னும் அமைப்பு உருவாயிற்று. நிலவுடைமையும் திருமணம் என்பதன் தோற்றத்திற்குக் காரணியாக அமைந்தது. நிலத்தில் பயிர் செய்யவும் காக்கவும் ஆள் துணையாகவும் பயன்பட்டவர் காலப்போக்கில் ஒருவருக்கொருவர் உடைமை எனக் கருதப்பட்டார். இதனால் மக்கட் செல்வம், குழந்தைச் செல்வம் என்று வழங்குகின்ற வழக்கம் உருவானது. பின்னர் திருமணம் என்பது கட்டாயம் என்ற நிலை சமூகத்தில் உருப்பெற்று வலுப்பெற்றது.

மணத்தின் தோற்றம் குறித்துத் தொல்காப்பியர்,

“பொய்யும் வழுவும் தோன்றிய பின்னர்

ஐயர் யாத்தனர் கரணம் என்ப" (தொல்காப்பியம், நூற்பா.1091)

என்று கூறுகின்றார். இதன் பொருள் பற்றிப் பல்வேறு கருத்துக்கள் நிலவி வந்தாலும் உலகியல் வழக்கில் காதல் வாழ்வில் பொய் தோற்றம் பெற்றதும் மூத்தோர் கரணம் (சடங்கு) என்ற ஒரு கட்டுப்பாட்டினை ஏற்படுத்தினர் என்னும் கருத்து ஏற்றுக்கொள்ளக் கூடியதாக உள்ளது (Tholkapiyam, 2008).

\section{மணமுறைகள்}

மக்கள் வாழ்க்கை முறையில் பலவிதமான மாற்றங்கள் ஏற்படுவது போன்று மணமுறைகளும் மாற்றம் பெற்றன.

"அன்பொடு புணர்ந்த ஐந்திணை மருங்கில்

காமக் கூட்டம்" (தொல்காப்பியம் - களவியல் 1)

எனப்படும் களவு மணத்தை விளக்குங்கால், “மறையோர்தே எத்து மன்றல் வகை எட்டு" என்று கூறியுள்ளார் தொல்காப்பியர்.

உரையாசிரியர்கள் இவற்றை முறையே, பிரம்மம், பிரசாபத்தியம், ஆரிடம், தெய்வம், காந்தருவம், அசுரம், இராக்கதம், பைசாசம் என்பதாக இனஞ்சுட்டியுள்ளனர். மேலும் சங்க காலத்தில் களவு மணம், தொன்றியல் மரபின் மணம், பரிச மணம், சேவை மணம், திசைக்கலப்பு மணம், ஏறுதழுவிய மணம், மடலேறி மணம், போர் நிகழ்த்தி மணம், துணங்கையாடி மணம், முகப்பேறு மணம் எனும் பத்து வகையான மணமுறைகள் இருந்தமையும் அறியமுடிகிறது.

ஒருதார மணம், பலதார மணம், பல கணவ மணம், குழு மணம், கொள்வினை மணம், பரிமாற்ற மணம், உடன்போக்கு மணம், வலிந்த மணம், சேவை மணம், அநுலோமம், பிரதிலோமம் போன்ற மணங்களைக் குறித்துக் கலைக்களஞ்சியம் விளக்கமளிக்கின்றது. மணமுறையின் தன்மையினை அடையாளம் காண அவரவர் திருமணமுறைகளைப் பற்றிய பாடல்களும் சடங்குமுறைகளும் துணை செய்கின்றன (Kalaikalanjiyam, 1961).

ஏலகிரி மலைவாழ்ப் பழங்குடிகளிடையே திருமணக் கட்டுப்பாடுகளும் காணப்படுகின்றன. பெரும்பாலும் காதல் திருமணங்களை இவர்கள் ஆதரிப்பதில்லை. சில வேளைகளில், தங்கள் இனத்துக்குள்ளே காதல் செய்வோராயின் திருமணத்திற்கு ஒப்புதல் அளிக்கின்றனர். மாறாக, மலைவாழ் மக்கள் சமவெளிப் பகுதியில் வாழும் மக்களிடையே பெண் கொடுப்பதுமில்லை; பெண் எடுப்பதும் இல்லை என்பது குறிப்பிடத்தக்கது. இக்கட்டுப்பாட்டினை மீறும்பட்சத்தில் ஊர் சபையைக் கூட்டி, மீறும் குடும்பத்தினரை ஊரை விட்டுத் தள்ளி வைப்பதுடன் அபராதத் தொகைகளை விதித்துக் கோவில் நற்காரியங்களுக்குப் பயன்படுத்தும் வழக்கத்தைக் கொண்டுள்ளனர். 
ஏலகிரி மலைவாழ் மக்களின் திருமணப் பாடல்கள்

இந்தியாவில் ஏறத்தாழ 427 பழங்குடி இன மக்கள் வாழ்கின்றனர். தமிழகத்தில் மக்கள் தொகை விகிதத்தில் 3.5 சதவீதம் பழங்குடிகள் உள்ளனர். ஏலகிரி மலையில் வாழும் இனக்குழுக்களை இருளர், காராளக்கவுண்டர், இந்துமலையாளிகள் என மூன்று வகைகளாகப் பிரிக்கலாம். ஏதோ சில காரணங்களால் மலைகளில் தற்போது குடியேறி வாழ்பவர்களை பழங்குடிகள் எனக் கூறி அவர்களை இப்பட்டியலில் இணைத்து விடுதல் இயலாது.

திருப்பத்தூர் மாவட்ட ஏலகிரிமலைப் பகுதியில் உள்ள பதினான்கு கிராமங்களிலும் வழங்கும் திருமண நிகழ்ச்சிகளையும் அதில் பின்பற்றப்பெறும் பல்வேறு சடங்குகளையும் மிகச் சிறப்பாகக் குறிப்பிடுகின்றன. அதில் திருமணச் சடங்கு பாடல்களும் ஒன்று. மணமக்களை வாழ்த்தும் பாடல்கள் கருத்தாழம் உடையவை.

“நாளது குறித்து

நல்விருந்துண்டு

வாழ்வது மங்கைக்கு

மனமது குளிர்த்து

நாளைத் திங்கள்

நற்கலி யாணம்"

என்று தொடங்கும் பாடல் திருமண நிகழ்ச்சிகளை முறையாகக் குறிப்பிடுகின்றது.

"பிடிபிடி வெற்றிலை

பிடி தனிப் பிடித்துப்

பாக்கு வெற்றிலை

பலபேர்க்குக் கொடுத்து

அன்னமும் தேனும்

அனைவோர்க்கும் கொடுத்து"

என்பதாகத் தொடர்கின்றனர். மேலும்,

"மிக்க மாப்பிள்ளையை

விரிதனில் இருத்தி

பெரிய கலத்தில்

பிரபல மாக

உரிய சாமை அரிசியும் உப்பும்

ஓரு மூன்று வள்ளம்

முக்காலி தன்னில்

முகூர்த்தமாய் வைத்து

பாரிய வெல்லம்

பாக்கு வெற்றிலையும்

வரிசை குறையாமல்

மதிப்புடன் வைத்து" 
என வரும் பாடல் திருமண உறவுமுறைப் பற்றியும் நடைபெறும் சூழலையும் எடுத்து இயம்புகிறது. பண்பாடு, நம்பிக்கை, ஆண் - பெண் உறவுமுறை போன்றவற்றைக் கூறுவதாக இப்பாடல் அமைகின்றது.

மணமகன் - மணமகள் உறவு முறை

“தன் அமர் ஆயமொடு நன்மண நுகர்ச்சியில்

இனிது ஆம் கொல்லோ தனக்கே பனிவரை

இனக் களிறு வழங்கும் சோலை

வயக்குறு வெள்வேல் அவர் புணர்ந்து செலவே" (ஐங்குறுநூறு.379)

இப்பாடல் சுற்றத்தாரோடு திருமணம் நடந்தேறும் முறையைக் கூறுகிறது. மேலும் சுற்றத்தின் துணையுடன் நிகழ்த்தப்படும் மணமானது மதிக்கப் பெறுவதாக அமைகிறது. ஏலலகிரி வாழ்ப் பழங்குடி மக்கள், தங்களுடைய இனத்தைச் சார்ந்த தங்களின் நெருங்கிய உறவுகளாக மதிக்கப்படுகின்ற தாய்மாமன் மகள், அத்தை மகள், அக்கா மகள், மண உறவு உள்ளவர்களாக இருப்பின் அதே சாதியைச் சேர்ந்த வேற்றுக் குலத்தில் திருமணம் செய்வதும், செய்து கொடுப்பதுமாகிய மரபான வழக்கங்களை உடையவர்களாக இருக்கின்றனர். முந்தைய காலத்தில், இருளர் இன ஆண் இருளர் இனப் பெண்ணைத் திருமணம் முடிக்க பெண் வட்டிற்குச் சென்று சில காலம் வேலை பார்க்க வேண்டும். பெற்றோருக்குப் பிடித்திருந்தால் மணம் முடிப்பர். பெண்ணை வலுக்கட்டாயமாகப் புணர்ந்து, குடும்பம் நடத்தி குழந்தை பெற்ற பின் அப்பெண் பிரிந்து செல்லலாம் அல்லது அந்த ஆணுடனே வாழலாம் எனும் வழக்கமும் இருந்துள்ளது.

\section{கடவுள் வழிபாடு}

திருமணத்திற்குப் பின்பு குடும்ப வாழ்வை ஏற்கும் மணமக்களின் எதிர்கால வாழ்வு சிறப்பாக அமைய வேண்டும் எனவும் அவர்கள் வாழ்வில் எவ்விதமான குறைகளும் இடையூறுகளும் இன்றிப் பிள்ளைப்பேறு கிடைக்கவேண்டும் என்றும் வேண்டிக்கொண்டு, திருமணத்திற்கு முன்பே ஊர்தெய்வங்களுக்கும், குலதெய்வங்களுக்கும், பொங்கல் வைத்து வழிபட்டு வருகின்ற மரபை இப்பழங்குடி மக்களிடையே காணமுடிகின்றது.

\section{பரிசம் போடுதல்}

ஒத்த குலம் சார்ந்தும் பொருளியல் அடிப்படையிலும் சமநிலைபெற்று இன்றைய திருமணம் நடைபெறுவது போலவே பண்டைய திருமணமும் நடைபெற்றது. இதனைக் குறித்துத் தொல்காப்பியர்,

“கற்பெனப் படுவ கரணமொடு புணர

கொளற்குரி மரபின் கிழவன் கிழத்தியைக்

கொனக்குரி மரபினோர் கொடுப்பக் கொள்வதுவே" (தொல்காப்பியம் பொருள் - 140)

என்று குறிப்பிடுகின்றார். இதைப்போலவே இம்மலைவாழ் மக்களின் திருமண முறை அமைவதைக் காண முடிகிறது. பெண்வீட்டாருக்கும் ஆண்வீட்டாருக்கும் இடையே ஏற்படுகின்ற திருமண ஒப்பந்த நிகழ்ச்சியினைப் 'பரிசம் போடுதல்', ‘நிச்சய தாம்பூலம்', 'பரியக்கட்டு' என்று பல்வேறு சொற்கள் வழக்கில் இ இருந்து வருகின்றது. பிற்காலத்தில் பரியம் என்பது வரதட்சணையாக பிற சமுதாயங்களில் வழங்கப்பெறுகின்றது. பரிசத்தொகை வழங்கும் வழக்கம் இப்பழங்குடிகளிடையே இருக்கும் சிறப்பம்சமாக விளங்குகிறது. ஆண்தான் பெண்ணுக்குப் பரிசம் போட்டுப் பெண் எடுக்க வேண்டும் என்பது இப்பழங்குடிகளின் வழக்கமாகும். ஒரு குறிபிட்ட நாளில் நிச்சயம் (நிச்சயதார்த்தம்) வைத்துக்கொள்ளலாம் என்று முடிவு செய்து, மாப்பிள்ளை வீட்டார் அந்த நாளில் தன் உறவினர், ஊர்ப் 
பொதுமக்கள் அனைவரையும் அழைத்துக்கொண்டு பெண் வீட்டிற்கு வருகின்றனர். பரிசத்தொகையைப் பேசி முடிவு செய்கின்றனர். அதில் ஒரு தொகையைப் பெண்ணுடைய தாய்மாமனுக்கு வழங்கவேண்டும் என்பது குறிப்பிடத்தக்கது. பரிசத் தொகையோடு சாமை, கேழ்வரகு, கம்பு, வரகு, ஆடு, மாடுகள் போன்றவற்றை பெண் வீட்டாருக்குக் கொடுத்துப் பெண் எடுக்கும் வழக்கமும் காணப்படுகின்றது.

\section{அழைப்பு முறை}

ஏலகிரி மலைவாழ் மக்கள் திருமணம் நடைபெறும் நாள் குறித்த செய்தியைத் தங்களுடைய உறவினர்களுக்கும் மற்றவர்களுக்கும் நேரில் சென்று வெற்றிலை, பாக்கு வைத்து மணமக்கள் வீட்டார் அழைக்கின்றனர். ஆனால், இன்றைய நவீன உலகில் பொருளாதாரத்தில் மிகவும் பின்தங்கியவர்களும் அழைப்பிதழ் அச்சிட்டுத் திருமணத்தை நடத்துகின்றனர்.

\section{மணப்பந்தல்}

பழங்குடி மக்கள் திருமணத்திற்காகப் அவரவர் இல்லத்திற்கு முன்பு பச்சை பந்தல் இடும் பழக்கம் காணப்படுகிறது. திருமணப்பந்தல் அமைப்பதற்கு முன்பு முகூர்த்தக்கால் நடும் பழக்கத்தினைக் கொண்டுள்ளனர். திருமணம் நடைபெறுவதற்கு மூன்று நாட்களுக்கு முன்பாக முகூர்த்தம் நடைபெறுவதற்குரிய இடத்தின் வட கிழக்கு மூலையில் பச்சை மூங்கில் நட்டு, அதற்கு மஞ்சள், சந்தனம், குங்குமம் வைத்து முதல் அரசாணைக்கால் நடுவது இவர்களின் மரபாக உள்ளது.

இல்லறத்தை ஏற்க இருக்கின்ற மணமக்களின் திருமணம் ஒரு பந்தலின் கீழ் நடப்பதால் பந்தலுக்கு எவ்விதத் தீங்கும் நேரிடாமல் நல்நிலையில் நடைபெறவேண்டும் என்று இறைவனை வேண்டி பந்தலுக்குரிய முதல் காலினை நட்டுப் பந்தல் அமைக்கின்றனர். திருமணத்திற்கு முதல் நாள் மணமக்களின் வீட்டின் முன்பு பந்தல் அமைத்து பலவகை மலர்கள் கொண்டு அலங்கரிக்கின்றனர். மணப்பந்தலில் அரச இலை, வேப்பிலை, மா இலை முதலானவை தோரணங்களகத் தொங்கவிடப்படுகின்றன. இச்செயற்கூறு, நாட்டுப்புற மருத்துவப் பண்பாட்டினோடு தொடர்புடையதாக அமைவதைக் காணமுடிகிறது.

பந்தலுக்கு நடுவில் மணமக்கள் அமருவதற்கு மரக்கட்டையால் ஆன மணமேடையை அமைத்து அதன் அடியில் நெல்லோ பச்சரிசியோ இடுவர்; இது அவர்கள் வாழ்வு நெல்லரிசிக்குக் குறைவின்றி அமையவேண்டும் என்கிற வளமைச் சடங்கினைக் குறிப்பதாக அமைகிறது. 'நிறை குடங்கள்' (சாலும் கரகம்) மணமக்கள் இ இருவரின் இல்லற வாழ்க்கை நிறைவானதாக அமையவேண்டும் என்பதற்கு அறிகுறியாக மணப்பந்தலில் மூன்று நிறை குடங்கள் மங்கலத் தோற்றத்துடன் மணமேடைக்கு அருகில் வைக்கப்படுகின்றன. இக்குடங்களில் மணமகளை ஆற்றுக்குச் செல்வித்து நீர் கொணர்கின்றனர். இந்நிகழ்வு வாழ்க்கையில் ஒரு பெண் குடும்பம் நடத்த வேண்டிய தகுதிப்பாட்டை பெற்றுவிட்டாள் என்பதைச் சடங்குகளின் வழி அனைவருக்கும் அறிவிக்கும் வண்ணம் உருவாகியதாகக் கருதமுடிகின்றது.

மணமகன் மணமகளுக்குத் தாலிகட்டி முடித்த பிறகு மணப்பந்தலில் வைக்கப்பட்டுள்ள முளைவந்த நவதானியங்களை, மேளதாளத்துடன் ஊரிலுள்ள ஆற்றங்கரைக்கு எடுத்துச் செல்கின்றனர். அங்கு பாத்திகள் அமைத்து மணமகள் நவதானியங்களை அள்ளித் தர மணமகன் வாங்கி பாத்தியில் விதைத்து வணங்குகின்றனர். மணப்பந்தலில் இருக்கும் அம்மியும் குளவியும், மணமக்கள் எதைச் செய்தாலும் ஒன்றாக இருந்து உறுதியுடன் செய்யவேண்டும் என்பதைக் குறிப்பதாக அமைகின்றது.

\section{நலுங்கு வைத்தல்}

திருமணத்திற்கு முன்பு மணமக்களுக்கு அவரவர் வீட்டில் நலுங்கு வைத்து விருந்து உபசரிக்கும் மரபு இப்பழங்குடி மக்களிடம் காணப்படுகிறது. நலுங்கு வைப்பது என்பது மணமக்கள் தங்களது இல்லற 
வாழ்வு தொடங்குவதற்கான ஆயத்தம் செய்யும் ஒரு சடங்கு ஆகும். இந்நிகழ்ச்சியால், நலுங்கு வைக்க வரும் சுற்றத்தாரோடு மணமகள் நெருங்கிப் பழகும் வாய்ப்பு ஏற்படுகிறது. மணமக்களை வீட்டின் நடுவில் கிழக்குத் திசை பார்த்தாற் போல் மரப்பலகையில் உட்கார வைத்து ஒருவருக்கொருவர் சந்தனம் கலந்த பல மூலிகைச் சாற்றைத் தலை முதல் உள்ளங்கால் வரை பூசும் நடைமுறை காணப்படுகின்றது.

\section{பெண் அழைப்பு}

திருமணத்திற்கு முதல்நாள் மணமகன் வீட்டார் உறவினர்களுடன் பச்சரிசி, மஞ்சள், வெற்றிலை, பாக்கு, உப்பு, புடவை போன்றவற்றைக் குலதெய்வங்களின் முன்பு வைத்துக் கும்பிட்டுப் பெண்வீட்டிற்குக் கொண்டு வருகின்றனர். பெண் வீட்டற்கு வந்ததும் அவர்களில் அரிசியை வைத்திருப்பவர்கள் மட்டும் வீட்டிற்குள் செல்கின்றனர். வீட்டில் பாயை விரித்துக் கொண்டுவந்த பொருட்கள் அனைத்தையும் வைக்கின்றனர். பிறகு, வெற்றிலை, பாக்கு இரண்டையும் எடுத்து ஊர்மக்கள் முன்னிலையில் நலங்கு வைக்கப் பயன்படுத்திய தட்டில் ஐந்து முதல் ஏழு சுற்றுவரை அடுக்குகின்றனர்.

\section{தாலி கட்டும் முன் செய்யும் சடங்கு}

மணமக்களின் வேட்டி, சட்டை, புடவை போன்றவற்றை மண நேரத்திற்கு முன்பாகத் தங்களின் குலதெய்வத்தின் முன் வைத்து வணங்கிய பின்னர் உடுத்துகின்றனர். மணமகன் குலதெய்வத்தை வழிபட்டு, தாய்மாமனுடன் மணவறையை மூன்று முறை சுற்றிவந்து, மணமேடையின் இடது பக்கமாக அமருகின்றார். மணப்பெண்ணும் இதே போல் அவளது தாய்மாமனோடு மணமேடைக்கு வந்து, தாய்மாமன் காலில் விழுந்து ஆசி பெற்று மணமகனின் வலது புறமாக அமருகின்றாள்.

\section{தாலி கட்டுதல்}

தேவநேயப் பாவாணர் மங்கள நாண் அணியும் வழக்கத்தைக் குறிப்பிடுகையில், "மணப்பருவம் வந்த பின்பு பலவகையிலும் ஒத்த இளைஞனும், இளைஞியும் தாமாகக் கூடியோ தம் பொற்றோரால் கூட்டப்பட்டோ கணவனும் மனைவியுமாக வாழ்வது ஆரிய வருகைக்கு முற்பட்ட பண்டைய தமிழ் மரபு. மணமானதற்கு அடையாளமாக மனைவியின் கழுத்தில் தாலி என்னும் மங்கள அணி அணியப்படும்" (Pillai, 1974 p.71) என்று விளக்குகின்றார். மணப் பொண்ணுக்கு மங்கள நாண் அணிவித்தல் நிகழ்வானது திருமணத்தின் உச்சபட்ச நிகழ்வாகத் தமிழ்ச் சமூகத்தில் காணப்படுகிறது. சிலப்பதிகாரப் புகார்க் காண்டத்தில் கோவலன் - கண்ணகிக்குமான திருமணத்தில்தான் முதன்முதலில் மங்கள அணி பற்றிய குறிப்புக் காணக்கிடைக்கின்றது.

தாலி கட்டும் வழக்கத்தில் இருக்கும் சடங்கியல் ரீதியான காரணங்கள் மட்டுமன்றி, சமூக அங்கீகாரத்திற்கான பிரச்சனையாகவும் மாறுவதைக் கே.கே.பிள்ளை, "மணம் பேசிவிட்டாலே திருமணம் முடிந்துவிட்டதாகக் கருதுவது தமிழ்பெண்களின் பண்பாடு. எனவே மகட் பேசியவன் இறந்ததும் தன் தாலி இழந்தவர்களாகக் கருதிகொள்பவரும் உண்டு. எனவே சங்ககாலத்திற்கு அருகிய நிலையில் தாலி அணியும் வழக்கம் இருந்ததே எனக் கருத இடமுண்டு. தாலி பற்றி குறிப்பிடும் முதல் கல்வெட்டு கி.பி.958ஆம் ஆண்டுக்கு உரியது." (Pillai, 1974 p.131-132) எனக் குறிப்பிடுகிறார்.

மணம் ஆவதன் அடையாளமாக மணமகளின் கழுத்தில் மங்களக் கயிறைக் கட்டுவதைத் ‘தாலி கட்டுதல்' என்று கூறுகின்றனர். இப்பழங்குடிகள் முன்பு மஞ்சள் கயிற்றை மட்டும் தாலியாகக் கட்டிக்கொள்வது மரபாக இருந்துள்ளது. இப்பழங்குடிகள் திருமணத்தை இவர்களின் முன்னோர்களே முன் நின்று நடத்துகின்றனர். அந்தணர் தாலி எடுத்துக் கொடுக்கும் வழக்கம் இல்லை; அதேபோல், வேத மந்திரங்கள் ஓதும் வழக்கமும் இல்லை. தாலி கட்டி முடித்தவுடன் மணமக்களுக்கு நெருங்கிய உறவினர்கள் தங்க நகைகளை அன்பளிப்பாகக் கொடுக்கின்றனர். பிறர் 'மொய்' எழுதுகின்றனர். திருமணத்திற்கு வந்தவர்களுக்கு அசைவ உணவு பரிமாறுவது வழக்கமாக உள்ளது. இவர்களின் 
திருமண நிகழ்ச்சியில் பன்றிக்கு முக்கியப் பங்குண்டு. திருமணத்தின்போது குலதெய்வத்திற்குக் காணிக்கை செலுத்தும் பொருட்டு, பன்றியைக் குத்தி ஊண் சமைத்து விருந்து வைக்கும் வழக்கம் இப்பழங்குடிகளிடையே இருந்து வருகிறது. இது, சமவெளிகளில் நிகழ்த்தப்படும் பொதுவான திருமண முறைகளிலிருந்து வேறுபட்டுள்ளதைக் காணமுடிகிறது.

ஏலகிரி மலைவாழ்ப் பழங்குடிகளில் பெரும்பாலானோர் திருமணத்தை மாப்பிள்ளை வீட்டிலும் நிச்சயத்தைப் பெண் வட்டிலும் நடத்துகின்றனர். சிலர் அவர்தம் குலதெய்வங்களான நாச்சியம்மா, நாச்சியப்பா கோவிலில் நடத்துகின்றனர்.

\section{மணமக்கள் விளையாட்டு}

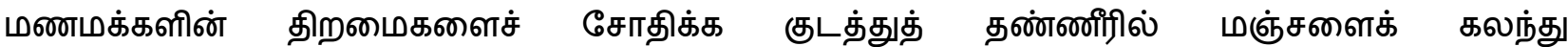
கண்புலனாகாத வண்ணம் செய்து அதில் மோதிரம், வளையல் போன்றவற்றைப் போட்டு எடுக்க வைத்தல்; குழந்தை போன்று பொம்மையைச் செய்து மாற்றி மாற்றி இருவரையும் வாங்கச் செய்கின்றனர். இத்தகைய விளையாட்டுகள் மூலம் மணமக்களுக்கு குடும்பம் நடத்துவது பற்றிய அச்சம் நீங்கி இருவரில் யார் அறிவு கூர்மை உள்ளவர் என்பதை அறிந்து கொள்ள முடிகிறது.

\section{மறுவீடு அழைப்பு}

திருமணம் முடிந்தவுடன் மணமக்களைப் பெண்வட்டிற்கு அழைத்து அசைவ விருந்து வைக்கும் முறையே மறுவீடு அழைப்பு (மறுமூசி) எனப்படுகிறது. பெண்வீட்டு உறவினர்கள் மணமக்களுக்கு ஆரத்தி எடுத்து வரவேற்கும் முறை உள்ளது. இந்த ஆரத்தி எனும் நிகழ்வை மணமகளுக்கு உடன்பிறந்த சகோதரிகளே எடுக்கின்றனர். இவ்வாறாக, திருமண நிகழ்வானது பண்பாட்டு ரீதிய சடங்குகளின் வழி அம்மக்களின் வளமைக்கான கூறாக உயிர்ப்போடு இருந்து வருகிறது.

\section{முடிவுரை}

ஏலகிரி மலைவாழ் இனக்குழுச் சமூக்கத்தின் எச்சமாக வாழும் இம்மக்கள் தங்கள் வாழ்வியலின் ஒரு அங்கமான திருமண நிகழ்வைத் தன்னைச் சார்ந்த சமூகச் சுற்றத்தோடு இணைந்து நடத்துகின்றனர். திருமணம் சார் சடங்குகள் இம்மக்களின் நாட்டுப்புறப்பாடல் வழி அறியமுடிகிறது. மனித இனம் சமூக ஒப்புதலோடு தம்முடைய பாலுணர்ச்சியின் வெளிப்பாட்டைச் செயல்பட அனுமதிக்கின்றது என்பதை இதன்வழி அறிய முடிகிறது. ஏலகிரி மலைவாழ் மக்களிடையே காணப்படும் திருமணச்சடங்குளில் ரிச்சர்டு எம். டார்சனின் பண்பாட்டுக் கோட்பாட்டினைப் பொருத்திப் பார்க்கையில், அவர்தம் பழமையான வளமைச் சடங்குகள் காலத்திற்கேற்ப நெளிவு கொடுத்து இன்னும் உயிர்ப்போடு அவர்களிடையே பின்பற்றப்பட்டு வருவதை இக்கட்டுரை வாயிலாக அறியமுடிகிறது. மேலும், நவீனப் போக்குவரத்துகள் காரணமாகவும், சுற்றுலாத் தளங்களின் பெருக்கம், காடழிப்புகள் காரணமாகவும் அவர்தம் பண்பாட்டுக் கூறுகள் பாதிப்புறுவதைத் தடுக்கும் பொறுப்பு தமிழ் கூறும் நல்லுலகிற்கு உண்டு என்பதையும் இக்கட்டுரை பதிவு செய்கிறது.

\section{References}

Kalaikalanjiyam (volume-8), (1961), Tamil Valarchi Kalagam, India.

Morgan, L.H., (1877), Ancient Society, United States.

Pillai, K.K., (1974), Thamizhaga Varalarum Makkalum Panpadum, Tamilnattu Paadanool Niruvanam, Chennai, India.

Richard M. Dorson, (1972), Folklore and Folk life - An Introduction, The university of Chicago press books.

Thayammal Aravanan, (1987), Uravumuraigal, Thamilkottam Pathipagam, India. 
Tholkapiyam, (2008), Ilampooranar Urai, Tamil University Maruthondri Pathipagam, Thanjavur, India.

\section{Information Providers}

Srinivasan (Indhu Malaiyali), Age - 33, Athanaur, Date:15.07.2020.

Papathi (Irular), Age - 45, Kottaiyur, Date:22.07.2020.

Amarasathi (Irular), Age - 58, Manchakollai Pudhur, Date:30.07.2020.

Vedi (Indhu Malaiyali), Age - 48, Thayalur, Date:05.08.2020.

Nachiyammal (Karala Gounder), Age - 67, Kaniyooran Vattam, Date: 06.08.2020.

Srinivasan (Karala Gounder), Age - 33, Athanaur, Date: 07.08.2020.

Murugan (Irular), Age - 40, Thayalur, Date: 07.08.2020.

Kuppu (Karala Gounder), Age - 58, Athanaur, Date: 15.08.2020.

Govindhasamy (Indhu Malaiyali), Age - 62, Nilavoor, Date: 17.08.2020.

Polliyanci (Indhu Malaiyali), Age - 55, Manchakollai Pudhur, Date: 17.07.2020.

\section{Funding: NIL}

Acknowledgement: NIL

Conflict of Interest: NIL

About the License:

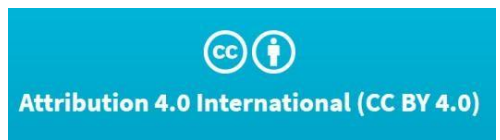

(C) The author 2021. The text of this article is licensed under a Creative Commons Attribution 4.0 International License 